\title{
INVERTING CONTROL, A NEW STRATEGY ON TIME DELAYED SYSTEMS
}

\author{
Ali Fuat Ergenc ${ }^{*}$, Hassan Fazelinia ${ }^{2}$, and Nejat Olgac ${ }^{2}$, \\ ${ }^{1}$ Istanbul Technical University, Electrical Eng. Control Div., Maslak, Istanbul, 34390 Turkey \\ ${ }^{2}$ University of Connecticut, Mechanical Engineering Department, Storrs, Connecticut 06269
}

\begin{abstract}
In this paper we introduce a new control strategy for linear time invariant (LTI) systems with a single time delay. The delay appears within the feedback control logic. Earlier research on this class of systems determines the delay intervals for stable operations, exhaustively, exactly and completely. The newly developed inverting control logic suggests a very practical procedure of reversing the sign of the feedback control gain, in order to enlarge the stable delay intervals. This gives some additional capabilities to control system designer. The sign inversion idea is based on a critical mathematical feature of LTI-TDS (Time Delay Systems) which was first recognized under a paradigm called the Cluster Treatment of Characteristic Roots (CTCR). Several example case studies demonstrate the practicality and the advantages of the new control law.

Copyright C 2002 IFAC
\end{abstract}

Keywords: Delay systems, Robust time delay systems, Infinite dimensional systems, Linear systems, Time invariant systems

\section{INTRODUCTION AND MOTIVATION}

Time delayed systems have been attractive for researchers for several decades (Cooke and van den Driessche 1986; Stepan 1989; Chen, Gu and Nett 1995; Gu and Niculescu 2001; Niculescu 2001). Earlier studies mainly focused on stability assessment problem. Infinite dimension of the linear time invariant (LTI) time delayed dynamics due to the transcendentally in the equations is notoriously complex to analyze. From the stability analysis angle, earlier investigations provided enormous insight. There is however, very limited control synthesis studies in the literature on time delayed systems, again mainly due to the notoriety of the problem (Niculescu 2001; Filipovic and Olgac 2002; Insperger and Stépán 2005). Author's group has contributed in this field, both from the stability analysis and the control synthesis aspects of the research (Olgac and Sipahi 2002; Olgac, Ergenc and Sipahi 2005; Sipahi and Olgac 2005; Olgac and Sipahi 2006; Sipahi and Olgac 2006; Fazelinia,

* Author was affiliated with University of Connecticut when the work was done.
Sipahi and Olgac; Ergenc, Olgac and Fazelinia, 2007). This manuscript presents a new synthesis prospective for control of the time delayed systems.

We consider a general class of LTI-TDS (linear timeinvariant, time delayed systems) where the delay is attributed to the feedback control:

$$
\dot{\mathbf{x}}=\mathbf{A} \mathbf{x}+\overline{\mathbf{B}} \mathbf{u}
$$

where $\mathbf{x}(n \times 1)$ is the state vector, $\mathbf{u}(m \times 1), m \leq n$, is the control, $\mathbf{A}$ and $\overline{\mathbf{B}}$ are matrices of appropriate dimensions. Taking a full-state feedback control law as $\mathbf{u}=\mathbf{K} \mathbf{x}(t-\tau)$, $\mathbf{K}(m \times n)$ being the feedback gain matrix and $\tau$ the delay which occurs in the feedback line, the system dynamics in (1) becomes:

$$
\dot{\mathbf{x}}=\mathbf{A x}+\mathbf{B} \mathbf{x}(t-\tau), \tau \in \mathfrak{R}^{+}, \mathbf{B}=\overline{\mathbf{B}} \mathbf{K}
$$

Author's group studied broadly, the stability robustness of this dynamics against uncertain (but constant) delays. (Olgac and Sipahi 2002; Olgac and Sipahi 2004; Sipahi and Olgac 2005; Olgac and Sipahi 2006; Sipahi and Olgac 2006) as well as the strategies for stabilizing (or disturbance rejecting) control (Olgac, Ergenc and Sipahi 2005). 
i) Our stability robustness investigations uncovered some interesting properties associated with the zeros of the characteristic equation corresponding to (2):

$$
C E(s, \tau)=\operatorname{det}\left(s \mathbf{I}-\mathbf{A}-\mathbf{B} e^{-\tau s}\right)
$$

The underlying paradigm which enables this analysis is called the Cluster Treatment of Characteristic Roots (CTCR). It uses two crucial properties to create an exact and exhaustive map of the stability robustness of (2) in the domain of $\tau \in \mathfrak{R}^{+}$. This result is interesting from the stability analysis as the CTCR methodology delivers the complete set of stable operating delays.

ii) We also developed and experimentally demonstrated a control strategy for (2), which we named the "Delay Scheduling Control" (Olgac, Ergenc and Sipahi 2005; Olgac, Sipahi and Ergenc, 2007). The philosophy in Delay Scheduling Control is that we intentionally increase the delay, $\tau$, i.e., we reschedule it, in order to improve the control performance. For instance, if the system is unstable with the present delay, we search for a larger delay which returns the system to stability. For some cases increasing the present delay can even improve disturbance rejection capability. In either objective one needs a crisp description of the delay regions that system operates stably, which we call the "stability pockets", especially for large delays. Since the CTCR procedure provides this information precisely, the control system designer has a better decision making ability as to which delay value, $\tau$, can be scheduled for stability.

The new control strategy we present in this paper is in a different direction altogether. It is primarily based on a unique feature of the imaginary spectrum of (3) (proposition II in (Olgac and Sipahi 2002)). Leaving the description of this feature to the following section we state the end result here. The idea is to switch the control gain $\mathbf{B}$ to $-\mathbf{B}$ (i.e., a single sign inversion) in (2) depending on the feedback delay, $\tau$. The expectation is that using $\mathbf{B}$ or $-\mathbf{B}$ in control we enlarge the stability pockets in the delay domain. This is an advantage, as the control designer now has broader range of selection for "delay scheduling" and potentially better control performance can be achieved using these extended regions.

The main motivation is this paper is to improve the control performance for a general class of LTI-TDS as in (1). And the novel contribution is in the suggestion of inverting the gain $\mathbf{B}$ to $-\mathbf{B}$. We explain the key properties of the system (1), in section II, along with the highlights of CTCR. In section III the new control logic is presented, as well as the mathematical reasoning behind it. Section IV entails a set of example case studies.

\section{AN OVERVIEW OF THE CTCR PROCEDURE}

Cluster Treatment of Characteristic Roots (CTCR) offers a unique approach to the TDS stability analysis. In essence, the CTCR method collects (i.e., "clusters") the infinitely many characteristic roots of the system into a small number of groups with some common clustering features. Very importantly, these clusters contain exhaustively, all the possible imaginary characteristic roots of equation (3). The key finding of the CTCR paradigm is that, the number of such clustered groups are proven to be small (therefore easy to handle) (Olgac and Sipahi 2002; Sipahi and Olgac 2003; Olgac and Sipahi 2006). Since the stability switching (from stable to unstable or vice versa) can only take place at an imaginary root, a systematic process naturally emanates from this point on revealing completely the stability outlook of the system, as we will describe later in the text.

The CTCR methodology also creates an explicit function, $N U$, for the number of unstable characteristic roots of the system, which is a function of $\tau$ only. This unique function declares the, so-called, stability pockets in $\tau \in \mathfrak{R}^{+}$domain, wherever $N U=0$. Interested reader is recommended to look at (Olgac and Sipahi 2002; Sipahi and Olgac 2003; Olgac and Sipahi 2006) for the details and relevant examples.

The most critical step in CTCR is to do with the determination of the "clustering features". What are they? How are they found? The answers to these questions are based on two propositions, which were not recognized until the authors' first publication (Olgac and Sipahi 2002). That publication offers an interesting structure and discipline guiding the imaginary root formations of the system. They are stated below without proofs.

Proposition I:The time-delayed dynamics (2) can exhibit only a bounded number of purely imaginary characteristic roots $\pm \omega_{k} i, k=1 \ldots M$ for all possible $\tau \in \mathfrak{R}^{+}$. This number, $M$, is upper bounded by $n^{2}$ (Sipahi and Olgac 2006). These imaginary characteristic roots may be single or multiple roots. Nevertheless there are infinitely many occurrence of imaginary root crossings at each of these $\omega_{k}$ 's for $M$ sets of time delays with infinite members. These delays, in each set, are equidistantly spread with $\Delta \tau_{k}=2 \pi / \omega_{k}$ in between, $\left(\tau_{k \ell}, k=1 \ldots M, \quad \ell=0,1,2 \ldots\right)$ starting from $\tau_{k 0}>0, k=1 \ldots M$.

In short, equation (2), when $\mathbf{A}, \mathbf{B}$ are fixed, has countably infinite characteristic roots, for a given delay, $\tau$. Call this infinite set of roots, $\sigma(\tau)$, which is the spectrum of equation (3). If for a $\tau_{k 0}>0$ value, $0<\tau_{k 0}<2 \pi / \omega_{k}$, $\sigma\left(\tau_{k}\right) \cap C^{0}= \pm \omega_{k} i$ (i.e., there is an imaginary root), then the same root will appear at $\tau_{k 0}+2 \pi / \omega_{k} j, j=0,1,2 \ldots$. In other words, $\quad \sigma\left(\tau_{k 0}+2 \pi / \omega_{k} j\right) \cap C^{0}= \pm \omega_{k} i, \quad j=0,1,2 \ldots$. will also hold. More importantly, the total number of such $\tau_{k 0}$ delays (each resulting in an imaginary spectrum), $M$, is bounded by $n^{2}$ (Sipahi and Olgac 2006; Ergenc, Olgac and Fazelinia In print, 2007). Exhaustively finding this small set of $\tau_{k 0}$ 's will "cluster" the delay values of interest for stability switchings.

Proposition I distinguishes the $M$ sets of $\tau$ values, each one 
of which yields at least a pair of imaginary roots. The exact values of $\tau$ that correspond to these potential stability switching points $\left(\tau_{k \ell}, k=1 \ldots M, \ell=0,1,2 \ldots\right)$ are needed completely. Notice that these time delays form an array with dimensions of $M \times \infty$ because of the above explained periodicity of $2 \pi / \omega_{k}$. Nevertheless they can be grouped into $\mathrm{M}$ distinct clusters each of which is designated by a root crossing point, $\omega_{k} i$. These crossing points are the $1^{s t}$ identifiers of the root clusters. In other words, the system (2) may be resonant at $M$ frequencies at the most, and these $\omega_{k}$ 's can be calculated exhaustively. How to find the rootcrossing frequencies, $\omega_{k}, k=1 \ldots M$, is a complex task on its own. The previous publications of the authors utilized an interesting Möbius class transformation, that is called the Rekasius substitution (Olgac and Sipahi 2002). We also published a dedicated comparison study on this task (Sipahi and Olgac 2006). We wish to stress that this question is a topic of on-going research.

\section{Proposition II: Root tendency invariance property}

The infinitely many characteristic roots which cross the imaginary axis at any one of the $\mathrm{M}$ locations for different (and periodically separated) delay values have a unique root sensitivity (either from stable to unstable, or vice versa) for increasing values of $\tau$. This root sensitivity is represented by root tendency, which is defined as

$$
R T_{k}=\operatorname{sgn}\left[\operatorname{Re}\left(\frac{\partial s}{\partial \tau}\right)\right]_{\substack{s=\omega_{k} i \\ \tau=\tau_{k \ell}}}, k=1 \ldots M, \ell=0,1,2 \ldots
$$

$R T_{k}$, is invariant with respect to the time delays that cause the particular root crossing.

What Proposition II implies is that at a designated root crossing point $\omega_{k} i$ (remember that, there are only $M$ of them), for all the equidistant and countably infinite time delays, the roots have a unique crossing direction. This unique feature $R T_{k}$ is called the $2^{\text {nd }}$ identifier of the root clusters. Those crossing points $\omega_{k} i$ with $R T_{k}=+1$ are clustered as destabilizing vs. those with $R T_{k}=-1$ as stabilizing. In short, the $M$ imaginary roots can cross either in $M$ stabilizing or destabilizing directions, as the delay $\tau$ is scanned from 0 to $\infty$ (keeping in mind that, there are infinitely many crossings at each $\omega_{k} i$ ).

The CTCR methodology deploys these two propositions for clustering the characteristic roots, as described in (Olgac and Sipahi 2002; Sipahi and Olgac 2003) generating a stability table. This table is sorted in ascending values of $\tau_{k \ell}$ $(k=1 \ldots M, \ell=0,1 \ldots)$. The root cluster identifiers, the crossing frequencies $\left(\omega_{k}\right)$ and the respective root tendencies ( $R T_{k}$ ), can also be given on the table, along with the number of unstable roots $(N U)$ in all the intervals of $\tau$. The intervals of $\tau$ where $N U=0$ are obviously the stable regions. Such regions are exhaustively determined with their exact bounds in $\tau$ domain.
Just to assist the reader, some highlights from (Olgac and Sipahi 2002) are given here: The quantity of $N U$ is a function of delay $\tau$ and it is explicitly given in the following form:

$$
N U(\tau)=N U(0)+\sum_{k=1}^{m} \Gamma\left(\frac{\tau-\tau_{k 1}}{\Delta \tau_{k}}\right) \cdot U\left(\tau, \tau_{k 1}\right) \cdot R T_{k}
$$

where $N U(0)$ is the number of unstable roots when $\tau=0, U\left(\tau, \tau_{k 1}\right)$ is a step function in $\tau$ with the step taking place at $\tau_{k 1}$

$$
U\left(\tau, \tau_{k 1}\right)= \begin{cases}0 & \text { for } 0<\tau<\tau_{k 1} \\ 1 & \text { for } \tau \geq \tau_{k 1}, \omega=0 \\ 2 & \text { for } \tau \geq \tau_{k 1}, \omega \neq 0\end{cases}
$$

$\Gamma(x)=$ Ceiling function of $x, \Gamma$ returns the smallest integer greater than or equal to $x$

This expression $N U(\tau)$ requires only the knowledge of the following:

i) $\mathrm{NU}(0)$

ii) $\tau_{k 1}, k=1 . . M$, the smallest $\tau$ values corresponding to each $\omega$ ( $M$ of them) as per Proposition I

iii) $\Delta \tau_{k}=2 \pi / \omega_{k}, k=1 . . M$

iv) $\left.R T\right|_{k}, k=1 . . M$ the invariant root tendencies, as per Proposition II.

This stability analysis methodology is exciting because it reveals the stability pockets entirely. Such information can be used as a "delay scheduling" tool. Assume that the state information is available to the controller with a particular time delay, which makes (2) unstable. The control logic may still be used maintaining stability, simply by imposing further prolonged delay on purpose, until the total delay reaches the next stable region. This is proposed as an intelligent management of time delay or a "delay scheduling" strategy, which gives the controller a unique ability. This control logic is in contrast to the well-known "gain scheduling" procedure. The new control strategy takes it from this point on.

\section{NEW CONTROL STRATEGY: "INVERTING CONTROL"}

The premise is to enlarge the stable operating delay intervals simply by selecting the feedback control gains as $\mathbf{B}$ and -B depending on the existing delay. The reason for this suggestion is as follows: according to the proposition II in CTCR a destabilizing characteristic root crossing for a delay setting, say $\tau_{0}$, repeats itself at $\tau_{0}+2 \pi k / \omega, k=1,2, \ldots$ infinity many times. Again all of theses root crossings take place at $\omega i$ with $R T=+1$. The delay values corresponding to these destabilizing crossings are periodically spaced with $2 \pi / \omega$. If we expect any stable region in between $\tau_{0}$ and $\tau_{0}+2 \pi k / \omega$ there has to be a stability crossing in that interval (i.e., with $R T=-1$ ) say at $\tau_{0}+\Delta \tau$, where $0<\Delta \tau<2 \pi / \omega$. The idea of increasing the delay artificially by $\pi / \omega$ could bring the delay value exactly in the middle of 
two successive destabilizing crossings. Obviously this selection does not guarantee stability but an interesting practicality appears. If one looks at the new characteristic equation (with $\tau \rightarrow \tau+\pi / \omega$ replacement):

$$
C E\left(s, \tau+\frac{\pi}{\omega}\right)=\operatorname{det}\left(s \mathbf{I}-\mathbf{A}-\mathbf{B} e^{-\left(\tau+\frac{\pi}{\omega}\right) s}\right)=0
$$

one can see that equation (5) has $s=\omega i$ as a root if $\mathbf{B}$ is replaced by $-\mathbf{B}$. It is trivial to prove that is such a case (5) and (3) become identical, thus they will share all the zeros. Conversely, if one substitutes $\mathbf{B}$ with $-\mathbf{B}$ in (3), the new system will exhibit imaginary crossings of $\omega i$, for the delay value of $\tau+\pi / \omega$ instead of $\tau$. That is, if $\dot{\mathbf{x}}=\mathbf{A x}+\mathbf{B} \mathbf{x}(t-\tau)$ has a characteristic root at $\omega i$ for delay $\tau$, with $R T=+1$, $\dot{\mathbf{x}}=\mathbf{A} \mathbf{x}-\mathbf{B} \mathbf{x}(t-\tau)$ has the same characteristic roots for delay $\tau+\pi / \omega$, with again the $R T=+1$. This property holds for all $\langle\tau, \omega\rangle$ occurrences, where the notation $\langle\bullet, \bullet\rangle$ implies that the first argument (i.e., the delay) causes a crossing frequency of the second argument. In essence $\mathbf{B} \rightarrow-\mathbf{B}$ inversion simply translates all the root crossing values of the delay by one half of the corresponding period $2 \pi / \omega$. Expectation is that the stable/unstable regions will also be translated so the if a delay value $\tau$ is unstable for the control gain matrix $\mathbf{B}$, it may now be stable when $-\mathbf{B}$ is used instead.

Since CTCR explicitly, exactly and exhaustively declares the stability distribution in the delay space one can efficiently obtain the superimposed stability tableau for $\mathbf{B}$ and $-\mathbf{B}$ control gains. The user can easily select either $\mathbf{B}$ or $-\mathbf{B}$ as the control gain matrix so that the stability will be assured. Obviously there will be delay values for which either B nor -B control law would render stability. Nevertheless the stable operating delay intervals for $\mathbf{B}$ and $-\mathbf{B}$ will be guaranteed to complement each other by enlarging the delay intervals of stability. This enlargement is the primary benefit of the Inverting Control logic. Since it involves nothing other that a sign inversion it is very practical. In the following section we present some case studies to illustrate the main idea behind this control strategy..

\section{CASE STUDIES}

We demonstrate the effectiveness of "Inverting Control" logic over two example case studies.

4.1 Case 1: Consider a single-degree-of-freedom system (such as a simple pendulum in (Olgac, Ergenc and Sipahi 2005)) which is controlled using a time delayed PD (proportional and derivative) feedback law. The corresponding system and control matrices are:

$$
\mathbf{A}=\left[\begin{array}{cc}
0 & 1 \\
-58.96 & -1.46
\end{array}\right], \overline{\mathbf{B}}=\left[\begin{array}{c}
0 \\
13.56
\end{array}\right], \mathbf{K}=\left[\begin{array}{ll}
1 & 0.1
\end{array}\right](6)
$$

and the characteristic equation

$$
C E(s, \tau)=s^{2}+1.458 s+58.957+(1.356 s+13.560) e^{-\tau s}=0
$$

The stable interval of delay can be obtained via CTCR using the implicit function $N U(\tau)$ (Number of Unstable roots) in (4). It imparts the stability intervals in $\tau$ domain as follows:

\begin{tabular}{||c|c||}
\hline $\begin{array}{c}\text { Stability } \\
\text { pockets }\end{array}$ & $\tau$ range \\
\hline 1 & {$\left[\begin{array}{ll}0, & 0.17\end{array}\right]$} \\
\hline 2 & {$[0.46,0.92]$} \\
\hline 3 & {$[1.38,1.66]$} \\
\hline 4 & {$[2.31,2.40]$} \\
\hline
\end{tabular}

Table 1. Stability table for the system in (7)

When we deploy the "inverting control" logic (by simply changing the sign of $\overline{\mathbf{B}}$ ) the new characteristic equation becomes:

$$
\begin{aligned}
\overline{C E}(s, \tau) & =\operatorname{det}\left(s \mathbf{I}-\mathbf{A}+\mathbf{B} e^{-\tau s}\right) \\
& \left.=s^{2}+1.458 s+58.957-(1.356 s+13.560)\right) e^{-\tau s}=0
\end{aligned}
$$

Again CTCR generates the stability tableau as

\begin{tabular}{||c|c||}
\hline $\begin{array}{c}\text { Stability } \\
\text { pockets }\end{array}$ & $\tau$ range \\
\hline 1 & {$\left[\begin{array}{ll}0, & 0.54\end{array}\right]$} \\
\hline 2 & {$\left[\begin{array}{ll}0.92, & 1.29\end{array}\right]$} \\
\hline 3 & {$\left[\begin{array}{ll}1.85, & 2.03\end{array}\right]$} \\
\hline
\end{tabular}

Table 2. Stability table for (6) with "inverting control"

The overlay of Table 1 and 2 is depicted in Fig. 1 which shows the advantage of mixing $\mathbf{B},-\mathbf{B}$ controls for different values of delays. The system is controlled in this mode to expand the range of delay for which the stability is assured.

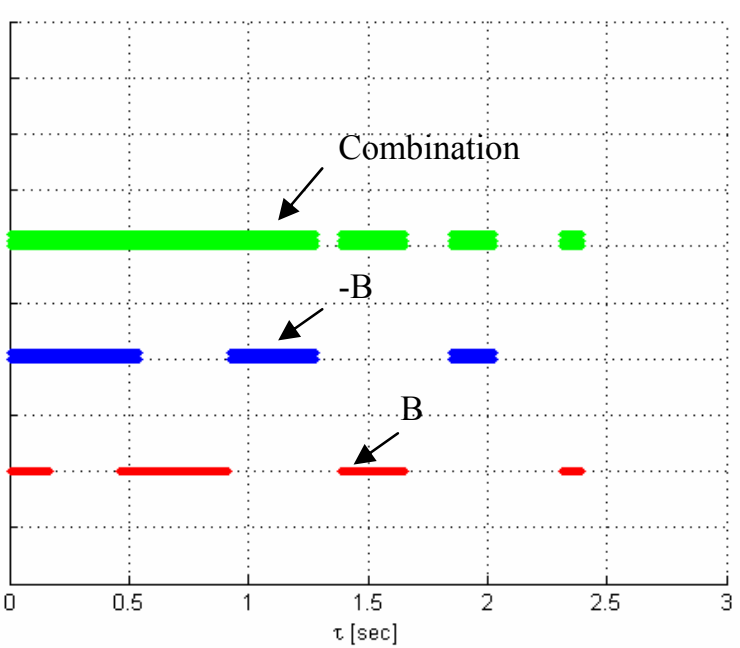

Figure 1. The stable operating intervals of delay B (red, thin), -B (blue, thicker), combined (green, thickest) 
Just to give an idea to the reader on the numerical efficiency: the Tables 1 and 2 are obtained in $1.8 \mathrm{sec}$ of CPU time (on a Pentium(R) 4 CPU $3 \mathrm{GHz}$ with $1 \mathrm{~GB}$ of RAM).

4.2 Case 2: We take another example from (Olgac, Sipahi and Ergenc, 2007), using

$$
\begin{aligned}
\mathbf{A} & =\left[\begin{array}{cccc}
0 & 1 & 0 & 0 \\
-79.61 & -1.46 & 20.33 & 0 \\
0 & 0 & 0 & 1 \\
3.21 & 0 & -30.43 & -0.89
\end{array}\right] \\
\mathbf{B} & =\left[\begin{array}{cc}
0 & 0 \\
13.56 & 0 \\
0 & 0 \\
0 & 2.28
\end{array}\right], \mathbf{K}=\left[\begin{array}{cccc}
-1 & 0 & -0.1 & 0 \\
-0.1 & 0 & -3 & 0
\end{array}\right]
\end{aligned}
$$

This system represents two interconnected simple penduli with motors deploying the necessary control torques. The characteristic equations for $\mathbf{B}$ and $-\mathbf{B}$ (inverted control) are given as:

$$
\begin{aligned}
C E(s, \tau)= & s^{4}+2.35 s^{3}+111.35 s^{2}+115.44 s+2357.72 \\
& +\left(20.39 s^{2}+22.07 s+965.08\right) e^{-\tau s}+(92.37) e^{-2 \pi s}=0 \\
\overline{C E}(s, \tau)= & s^{4}+2.35 s^{3}+111.35 s^{2}-115.44 s+2357.72 \\
& -\left(20.39 s^{2}+22.07 s+965.08\right) e^{-\tau}+(92.37) e^{-2 \pi s}=0
\end{aligned}
$$

CTCR efficiently determines the stability outlook of Table 3. Again the combination of the stability tables for two settings $\mathbf{B}$ and $-\mathbf{B}$ are displayed in Figure 2. The advantage of the inverting control is obvious.

Just for the numerical efficiency point of view, we provide the CPU time needed to obtain the Table 3 as $2.3 \mathrm{sec}$.

\begin{tabular}{||c|c|c||}
\hline $\begin{array}{c}\text { Stability } \\
\text { pockets }\end{array}$ & $\tau$ range $(\mathbf{B})$ & $\begin{array}{c}\tau \text { range (-B): } \\
\text { inverting control }\end{array}$ \\
\hline 1 & {$[0,0.14]$} & {$[0,0.50]$} \\
\hline 2 & {$[0.51,0.85]$} & {$[0.57,0.68]$} \\
\hline 3 & {$[0.93,1.22]$} & {$[1.16,1.20]$} \\
\hline 4 & {$[1.81,2.23]$} & {$[1.28,1.76]$} \\
\hline 5 & {$[3.11,3.38]$} & {$[2.46,2.58]$} \\
\hline 6 & & {$[2.71,2.84]$} \\
\hline 7 & & {$[3.76,3.92]$} \\
\hline
\end{tabular}

Table 3. Stability tables for example case 2.

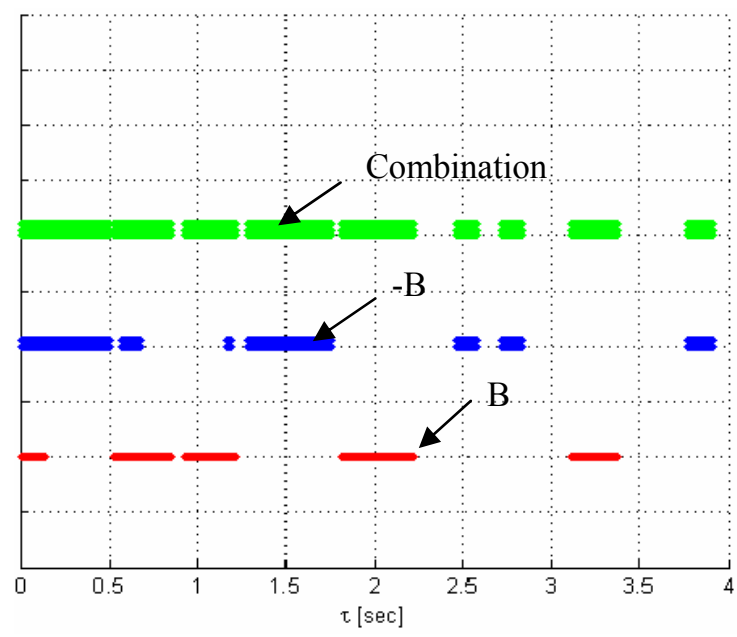

Figure 2. The stable operating intervals of delay example case 2. B (red, thin), -B (blue, thicker), combined (green, thickest)

\section{CONCLUSIONS AND FUTURE WORKS}

The "inverting control" is a control law to increase the stable operating intervals of the systems with feedback delays. The procedure takes advantage of the strength of a paradigm: Cluster Treatment of Characteristic Roots (CTCR). It is numerically very efficient, and the only critical change in the control structure is the sign reversal of the control matrix B for different delay values. This is a straightforward task. The ongoing work along the "inverting control" idea includes experimental validation of the method, and deployment of complex cases for higher order systems and with multiple independent delays.

\section{REFERENCES}

Chen, J., Gu, G. and Nett, C. N. (1995). "A new method for computing delay margins for stability of linear delay systems." Systems \& Control Letters 26: 107-117.

Cooke, K. L. and van den Driessche, P. (1986). "On Zeros of Some Transcendental Equations." Funkcialaj Ekvacioj 29: 77-90.

Ergenc, A. F., Olgac, N. and Fazelinia, H. (2007). "Extended Kronecker Summation for Determining the Kernel and Offspring of LTI Systems with Multiple Delays." SIAM Journal on Control and Optimization 46 (1): 143-155.

Fazelinia, H., Sipahi, R. and Olgac, N. (2007). "Stability Analysis of Multiple Time Delayed Systems Using 'Building Block' Concept." IEEE Transactions on Automatic Control 52(5):799 - 810.

Filipovic, D. and Olgac, N. (2002). "Delayed resonator with speed feedback-design and performance analysis." Mechatronics 12(3): 393-413.

Gu, K. Q. and Niculescu, S.-I. (2001). "Further remarks on additional dynamics in various model 
transformations of linear delay systems." $\underline{\text { IEEE }}$ Transactions on Automatic Control 46(3): 497-500.

Insperger, T. and Stépán, G. (2005). Act and wait control concept in force-controlled systems with discrete delayed feedback. Proceedings of ASME International Design Engineering Technical Conferences, Long Beach, California.

Niculescu, S.-I. (2001). Delay effects on stability, SpringerVerlag, Berlin.

Olgac, N., Ergenc, A. F. and Sipahi, R. (2005). "Delay Scheduling', For Stabilization in Multiple Delay Systems." Journal of Vibration and Control 11(9):1159-1172.

Olgac, N. and Sipahi, R. (2002). "An exact method for the stability analysis of time delayed LTI systems." IEEE Transactions on Automatic Control 47(5): 793-797.

Olgac, N. and Sipahi, R. (2004). "A Practical Method For Analyzing the Stability of Neutral Type LTI-Time Delayed Systems." Automatica 40: 847-853.

Olgac, N. and Sipahi, R. (2006). "An Improved Procedure in Detecting the Stability Robustness of Systems with Uncertain Delay." IEEE Transactions on Automatic Control 51(7): 1164 - 1165.

Olgac, N., Sipahi, R. and Ergenc, A. F. (2007). "'DELAY SCHEDULING', an Unconventional Use of Time Delay for Trajectory Tracking." Mechatronics. 17(4-5): 199-206

Sipahi, R. and Olgac, N. (2003). "Degenerate Cases in Using Direct Method." Transaction of ASME, Journal of Dynamic Systems, Measurement, and Control 125(2): 194-201.

Sipahi, R. and Olgac, N. (2005). "Complete Stability Map of Third Order LTI, Multiple Time Delay Systems." Automatica 41(9): 1413-1422.

Sipahi, R. and Olgac, N. (2006). "Stability Robustness of Retarded LTI Systems with Single Delay and Exhaustive Determination of Their Imaginary Spectra." SIAM Journal on Control and Optimization 45 (5): 1680-1696.

Sipahi, R. and Olgac, N. (2006). "A Unique Methodology for the Stability Robustness of Multiple Time Delay Systems." Systems \& Control Letters 55(10): 819825.

Stepan, G. (1989). Retarded Dynamical Systems: Stability and Characteristic Function. New York, Longman Scientific \& Technical,co-publisher John Wiley \& Sons Inc., US. 